\title{
Familie und Schule in der Adoleszenz: Ergebnisse qualitativer Längsschnittuntersuchungen zu Bewährungsanforderungen von der Grundschule bis zum Übergang in die Ausbildung bzw. das Studium
}

\author{
Mirja Silkenbeumer $\cdot$ Sven Thiersch
}

Eingegangen: 23. September 2020 / Überarbeitet: 14. Mai 2021 / Angenommen: 19. Mai 2021 / Online publiziert: 23. Juni 2021

(C) Der/die Autor(en) 2021

Zusammenfassung Der Beitrag stützt sich auf Erkenntnisse aus drei qualitativen Längsschnittstudien, in denen Heranwachsende und ihre Familien in relevanten Phasen der Schullaufbahn befragt und Bildungsverläufe aus subjekttheoretischer Sicht analysiert worden sind. Auf der Grundlage der übergreifenden Befunde dieser Untersuchungen wird argumentiert, dass sich Jugendliche in schulischen Bildungsverläufen, unabhängig davon, wie erfolgreich diese vollzogen werden und in welche sozialen Strukturen sie eingebettet sind, als Subjekte bewähren müssen. Sie sind in der Adoleszenz damit konfrontiert, sich gegenüber schulischen Anforderungen und bildungsbezogenen Delegationen in ihren Familien zu positionieren und den Bildungsverlauf in das werdende Selbst zu integrieren. Diese kaum erforschten und diskutierten subjektiven Modi, Bewährungsanforderungen zu gestalten, sind mit Selbst- und Fremdzuschreibungen im Kontext beziehungsdynamischer, intergenerational strukturierter Ablösungs- und Individuationsprozesse in Familie und Schule verbunden. Im Beitrag werden unterschiedliche adoleszente Bewährungsformen im Zusammenspiel von Familie und Schule typologisch ausgeleuchtet. Vor diesem Hintergrund differenzieren wir die bisherigen Erkenntnisse der Schul- und Bildungsforschung zur sozialen Hervorbringung schulbezogener Haltungen sowie zu Verläufen und Übergängen von Jugendlichen im Bildungssystem.

Schlüsselwörter Familie $\cdot$ Schule $\cdot$ Bildung $\cdot$ Adoleszenz $\cdot$ Subjekt $\cdot$ Bewährung

\footnotetext{
Prof. Dr. Mirja Silkenbeumer

Fachbereich Erziehungswissenschaften, Institut für Sonderpädagogik, Goethe-Universität Frankfurt I

Campus Westend, Theodor-W.-Adorno-Platz 6, 60323 Frankfurt am Main, Deutschland

Prof. Dr. Sven Thiersch $(\bowtie)$

Institut für Erziehungswissenschaft, Arbeitsbereich Bildungssoziologie und Sozialisationsforschung, Ruhr-Universität Bochum, Universitätsstraße 150, 44780 Bochum, Deutschland

E-Mail: sven.thiersch@rub.de
} 


\title{
Family and school in adolescence-results of qualitative longitudinal studies on probation from primary school up to the transition to training or university studies
}

\begin{abstract}
The article is based on results from three qualitative longitudinal studies in which adolescents and their families were interviewed at relevant stages of school careers. The educational pathways were analysed from a subject-theoretical perspective. Based on the overarching results of these studies, adolescents must prove themselves as social subjects in their school careers, regardless of how successfully their educational pathways are completed and in which social structures they are embedded. Adolescents are so confronted with the task of positioning themselves in relation to the school-related delegations in their families on one hand, and, on the other hand, of integrating their own educational process into their becoming self. These subjective probationary requirements, which have, so far, rarely been researched and discussed, are linked to an appropriation of school and a (re)addressing of the self in the context of relationship-dynamic, intergenerational structured processes of separation and individuation. This article examines different forms of adolescent probation in the context of family and school. On this background, we differentiate the previous results of school and educational research on the social production of school-related attitudes as well as on processes and transitions of adolescents in the educational system.
\end{abstract}

Keywords Family $\cdot$ School $\cdot$ Education $\cdot$ Adolescence $\cdot$ Subject $\cdot$ Probation

\section{Einleitung}

In den 1980er und 1990er-Jahren sind bereits zahlreiche Untersuchungen durchgeführt und Ergebnisse vorlegt worden, die das Verhältnis von Familie, Schule und Jugend in den Blick nehmen. Adoleszente Schüler/innen wurden als Akteure bestimmt, die schulische Anforderungen vor dem Hintergrund familial-biographischer Erfahrungen und adoleszenter Krisendynamiken eigensinnig bearbeiten. Dabei rückte das Zusammenspiel der interaktionslogisch different strukturierten Sozialisationsräume Schule und Familie hinsichtlich der Konstitution jugendlicher Subjektivität in den Fokus (z.B. Helsper et al. 1991; Fend 1997). Die „Schule als Entwicklungsraum“ für Jugendliche und kehrseitig den ,Entwicklungsaspekt als Bestimmungsstück pädagogischen Handelns“ sah etwa Fend (1997, S. 7) als zentrale Gegenstände für die erziehungswissenschaftliche Reflexion und sowohl in der Entwicklungspsychologie als auch in der Schulpädagogik bzw. -forschung damals vernachlässigt. Betrachtet man die Entwicklung in den sich inzwischen weit verzweigten Feldern der Bildungsforschung der letzten Jahre, gewinnt Fends damalige Analyse erneut an Aktualität: Denn die Entwicklung hin zu einer evidenzbasierten Bildungspolitik und -praxis und zu neuen Steuerungselementen führte zu einem verstärkten Interesse an Ergebnissen zum Output fachbezogener Kompetenzen und zur Optimierung der Lernmotivation und Leistungsbereitschaft von Schülern/innen (u. a. Pongratz et al. 2007). Fragen der Sozialität schulischen Lernens, der Hervorbringung und Ausformung 
bildungsbiographischer Selbstentwürfe und Positionierungen der Schüler/innen in der Adoleszenz aus einer subjekttheoretischen Perspektive sind in der bildungswissenschaftlichen Forschung insgesamt eher in den Hintergrund getreten.

Im folgenden Beitrag möchten wir, nach einem knappen Rückblick auf den schon angesprochenen Diskurs zur biographischen Be- und Verarbeitung des Schulischen im Verhältnis zu familialen Strukturen und Dynamiken in der Adoleszenz (2.), Ergebnisse aus drei, in der rekonstruktiven Bildungsforschung angesiedelten Projekten vorstellen (3.). In der komparativen Analyse dieser Untersuchungen hat sich uns die Kategorie der Bewährung zur Beschreibung adoleszenter Selbstpositionierung zwischen Familie und Schule erschlossen, die wir zunächst theoretisch bestimmen werden (4.), um dann die Befunde zu den Bewährungsanforderungen in jugendlichen Bildungsprozessen und -verläufen übergreifend zusammenzufassen (5.) und konzeptionell weitergehend zu bestimmen (6.). Ziel des Beitrags ist es somit, bewährungstheoretische Überlegungen als heuristisch instruktiv für die Rekonstruktion der fallspezifischen Dynamiken von Bildungsverläufen und adoleszenten Bildungsprozessen zu diskutieren und aus einer sozialisationstheoretisch fundierten mikroanalytischen Forschungsperspektive die Bedeutung des interaktiven Positionierungsund Aushandlungsgeschehens für die Bearbeitung schulischer Anforderungen in der Adoleszenz, wie wir es im Fazit resümieren werden, (wieder) deutlicher ins Bewusstsein von Bildungstheorie und -forschung zu rücken (7.).

\section{Schule in der Adoleszenz: Exemplarischer Rückblick auf den Diskurs}

Im Diskurs zum Zusammenhang von schulischer Sozialisation und Identitätsentwicklung standen in der Vergangenheit vor allem zwei Arbeiten aus zwei unterschiedlichen Paradigmen im Zentrum: Zum einen hat Franz Wellendorf im Theorierahmen der Psychoanalyse, des Strukturfunktionalismus und des Symbolischen Interaktionismus bereits Ende der 1960er-Jahre hervorgehoben (1974, S. 37 ff.), welche „Balanceleistungen“ Jugendliche in der Wahrung der sich formierenden ,,persönlichen Identität" angesichts der an sie herangetragenen Identitätszuschreibungen und -zumutungen der Institution bewerkstelligen müssen. Damit nahm er eine Fragestellung in den Blick, die sich gegenüber anderen sozialen Organisationen in besonderer Weise in der Schule stellt, ,da die schulischen Interaktionsprozesse unter der Bedingung einer noch relativ schwach ausgebildeten Ich-Identität der größeren Gruppe der Interaktionspartner (der Schüler/innen) abläuft“ (ebd., S. 49). Zudem arbeitete er heraus, in welcher Weise Rollenkonflikte, die aus der Ambivalenz von Eltern-KindInteraktionen und Schule resultieren, zur Identitätsbehauptung der Eltern beitragen. „Praktiken der Rollendistanzierung“ (Wellendorf 1969, S. 28) von Eltern können nach seiner Analyse wiederum , die Unsicherheit des jugendlichen Schülers bei seiner eigenen Identitätsfindung" verstärken (ebd., S. 30). Er wies so nicht nur auf die Bedeutung der Formierung und Möglichkeiten der Behauptung von Identität im Verhältnis zu sozialen und symbolischen Positionierungen hin, sondern auch schon auf die Bedeutung familial-generationaler Dynamiken und Relationen von Familie und Schule in schulischen Sozialisationsprozessen. 
Zum anderen verfolgte Helmut Fend in seinen Untersuchungen zur Ausbildung eines (Leistungs-)Habitus und der Identität bei Jugendlichen in der Schule als Erfahrungs- und Anforderungsraum - aus einer stärker empirisch geleiteten Perspektive das Erkenntnisinteresse, für diese Prozesse ,universale Entwicklungsperspektiven ebenso zu beachten wie interindividuell variierende Bewältigungsformen der Verarbeitung von Schule“ (Fend 1997, S. 8f.). Neue Formen der Selbstbeobachtung und -reflexivität erzeugen ,die Besonderheit der Entwicklung des Selbst in der Adoleszenz“, die eine „Differenz von Möglichkeit und Wirklichkeit, von Idealität und Realität sowie die von Innen und Außen, von Gezeigtem und Verborgenem“ auch in Bezug auf schulische Anforderungen analytisch akzentuiert (Fend 1994, S. 210). In der longitudinal angelegten, quantitative und qualitative Ansätze verbindenden Untersuchungsserie „Adoleszenz in der Moderne“ fokussierten er und seine Arbeitsgruppe diese Auseinandersetzung mit der Schule in der Adoleszenz im Verlauf vom 6. bis zum 10. Schuljahr im sozialen Kontext von Familie und Peers als „Mitkonstrukteure des Selbst“" (ebd., S. 201) sowie ihrer Einbettung in gesellschaftliche Modernisierungsprozesse. Gestützt auf entwicklungspsychologische, soziokognitive und handlungstheoretische Ansätze belegen die Befunde (ebd., S. 20), wie die reflexive, dynamische und von ko-konstruktiven Prozessen gerahmte Entwicklung, Bildung und Akzeptanz des Selbst in der Adoleszenz mit veränderten Ausprägungen der Leistungsbereitschaft verknüpft und diese Zusammenhänge dabei ,in hohem Maße kontext-abhängig“ (soziale Herkunft, Schulform) sind (Fend 1997, S. $252 \mathrm{f}$. und S. 335). Resümierend zeigen die Ergebnisse eine „Verflochtenheit von Leistung, Selbst und Sozialität“ als ,,individuelle Konfiguration der Bewältigung von Schule“ zwischen ,aktiv-erfolgsorientierter" und ,,abwehrend-vermeidender" Bearbeitung (ebd., S. 342).

Vor dem Hintergrund dieser zwei zentralen Arbeiten sind - vor allem hermeneutisch-rekonstruktive - Untersuchungen durchgeführt worden, in denen die subjektive Deutung, Bearbeitung und Integration von Erfahrungen und Ereignissen der Schulkarriere im biographischen und lebensweltlichen Gesamtzusammenhang von Jugendlichen im Zentrum stehen (Helsper et al. 1991; Nittel 1992; im Überblick Helsper 2008). Mit diesem theoretischen und inhaltlichen Zuschnitt auf die Sinndeutungen und Aushandlungsprozesse wird herausgearbeitet, welche Bedeutung schulisches Erleben für adoleszente Individuationsprozesse und die strukturell dynamischkrisenförmige Subjektwerdung aufweist (Combe und Helsper 1994, S. 107; im Überblick Kramer 2014) und welche Bildungsprozesse Jugendliche in der Gestaltung und Bewältigung der Schullaufbahn vollziehen. Daran anschließende Untersuchungen arbeiten das Zusammenspiel pädagogischer Generationsbeziehungen in Familie und Schule für die Eröffnung oder Begrenzung von Gestaltungsspielräumen für adoleszente Individuierung, den Bildungsverlauf und -prozess Jugendlicher heraus (Helsper et al. 2009), auch mit besonderem Fokus auf die Bedeutung intergenerationaler familialer Dynamiken für adoleszente Entwicklungs- und Bildungsprozesse sowie Bildungskarrieren von Jugendlichen mit Migrationsgeschichte (King 2009; King et al. 2011). Mit diesen Arbeiten sind generationale Ordnungen und intergenerationale Bildungsorientierungen in ihrer Relevanz für Prozesse der Subjektbildung und für Transformations- bzw. Reproduktionsprozesse des familialen Erbes in der Adoleszenz empirisch fundiert ausgeleuchtet und theoriegenerierendes Potenzial 
entfaltet worden. Letztlich ist es aber bislang ,nicht zur Etablierung einer breiten Theorie- und Forschungsströmung gekommen“ (Kramer 2014, S. 434), in der der Individuationsprozess der Schüler/innen und damit verbundene Fragen der Hervorbringung von Identität und einer Transformation des Habitus zwischen Familie und Schule im Zentrum steht.

\section{Jugendliche Bildungsprozesse und -verläufe zwischen Familie und Schule: Drei qualitative Längsschnittstudien}

In diesem Beitrag sollen die Ergebnisse aus drei qualitativen Längsschnittuntersuchungen zur Diskussion gestellt werden, die wir vor dem Hintergrund der jeweiligen heuristischen Rahmungen und forschungsmethodischen Zugriffe in Beziehung zueinander setzen und ausgehend von der Frage der adoleszenten Selbstpositionierung zu Bildungsprozessen und -verläufen zwischen Familie und Schule einer komparativen Metaanalyse unterziehen. In allen drei Untersuchungen steht das Erkenntnisinteresse im Zentrum, die adoleszente Aneignung und Bearbeitung schulischer Anforderungsstrukturen im Spannungsfeld von Transformation und Reproduktion zu rekonstruieren. Um das theoriegenerierende Potenzial der Forschungsergebnisse weiter entfalten zu können, hat sich dabei das Konzept der Bewährung als fruchtbar erwiesen. Insofern werden zunächst knapp die Projekte, nicht jedoch im Weiteren Einzelergebnisse und -fallstudien vorgestellt. ${ }^{1}$ Das Ziel der metaanalytischen Betrachtung ist vielmehr, die Ergebnisse der drei Längsschnitte zusammenzuführen und in der kontrastiven Sichtung übergreifende Erkenntnisse zu gewinnen. Der Bewährungsbegriff stand weder in der theoretischen Rahmung noch in der theoretischen Rückkopplung der Ergebnisse in den drei Studien im Zentrum. In der Komparation und Diskussion der Befunde treten die Bewährungsanforderungen und Formen ihrer Bearbeitung hervor, die mit Neu- und Umgestaltungen familialer und schulbiographischer Erfahrungskonstellationen verbunden sind. Eine besondere Berücksichtigung finden hierbei die Übergänge der jugendlichen Schüler/innen im Kontext biographischer, familialer und institutioneller Zusammenhänge. Im Längsschnitt sind dazu mehrfach qualitativ-narrative Interviews in relevanten Passagen der Schulkarriere an institutionellen (nach der Grundschule, Sek. I und II) und individuellen Übergängen (Klassenwiederholungen, Umzüge usw.) mit Jugendlichen und ihren Eltern durchgeführt worden.

Um die Spezifika der Transformation schulischer Orientierungen am Übergang und in der frühen und mittleren Adoleszenz zu betrachten, beziehen wir uns erstens auf die Längsschnittstudie „Erfolg und Versagen in der Schulkarriere - Ein qualitativer Längsschnitt zur biographischen Verarbeitung schulischer Selektionsereignisse“, welche aus einer praxeologisch-strukturtheoretischen Perspektive schulische Selektionserfahrungen und deren biographische Verarbeitung von 70 Heranwachsenden vom Ende der Grundschule bis zur 9. Klasse untersucht und diese mit der Dokumentarischen Methode auswertet (Kramer et al. 2009, 2013). Die Analysen zielen

\footnotetext{
${ }^{1}$ Die Autorin und der Autor des Beitrags waren als Projektleitung oder Projektmitarbeitende an diesen Studien beteiligt.
} 
auf eine Rekonstruktion von schulbezogenen Orientierungsrahmen bzw. Habitus und ihrer Transformation im Bildungsverlauf. In der Analyse dieser Transformation und Genese des Bildungshabitus zeigt sich in der Auswertung der Interviews ab Klasse 7, dass die primär in der Familie erworbenen habituellen Orientierungen in der Präadoleszenz unter verstärkten Bewährungsdruck geraten und Schüler/ innen erste Selbstpositionierungen gegenüber schulischen Anforderungen einnehmen. Diese Ergebnisse deuten wir so nicht nur als eine Passungsfrage von Familienund Schulmilieus im sozialen Raum, sondern auf der Ebene des Subjekts und seiner Positionierung zur Passung und zum eigenen Bildungsverlauf (Thiersch 2020a). Für die Erklärung von Habitustransformationsprozessen (z. B. bei einem Aufstieg) ist die Adoleszenzkrise als eine entscheidende Phase zu betrachten, weil sich die Jugendlichen zur Schule und zum familialen Erbe zunehmend positionieren müssen. Dies beinhaltet Potenziale sowohl für Krisen des Subjekts und Konflikte in Familie und Schule als auch für Habitustransformationsprozesse (Helsper 2014).

Zweitens kommt die Studie „Die Mühen des Aufstiegs: Fallrekonstruktionen zur familialen und bildungsbiografischen Dynamik erwartungswidriger Schulkarrieren“ in den Blick, in der bildungsbiographische Selbstpositionierungen von der mittleren bis zur Spätadoleszenz ${ }^{2}$ Gegenstand der Analyse sind. Aus subjekttheoretischer Sicht wird gefragt, wie ,erwartungswidrige“ Schulkarrieren und Bildungsaufstiege in der familialen Interaktion und den adoleszenten Selbstpositionierungen konstelliert sind. Die 34 Jugendlichen und ihre Familien werden von der 10. Klasse bis zur Ausbildung bzw. zum Studium wiederholt in leitfadengestützten Interviews befragt, die mit der Objektiven Hermeneutik analysiert werden (Silkenbeumer et al. 2017; Labede et al. 2020).

Schließlich rekurrieren wir drittens auf die laufende Untersuchung „Transformations(an)forderungen im Übergang zwischen Familie, Kinder- und Jugendpsychiatrie und Psychotherapie und (Klinik-)Schule in der Adoleszenz" (Trübas) (Becher und Schulmeister 2020; Silkenbeumer et al. 2020; Becher et al. 2021). Das Untersuchungsinteresse zielt hier auf die Identifikation von Potentialitäten und Begrenzungen in der Lebenslage psychosozialer Beeinträchtigung für adoleszente Bildungsprozesse und Bildungskarrieren im Zusammenspiel familialer, schulischer und medizinisch-therapeutischer Sozialisationsräume. Über drei Erhebungszeiträume geführte bildungsbiographische Interviews mit sieben psychiatrieerfahrenen Klinikschülern/

\footnotetext{
2 Wenn wir von einer frühen, mittleren und späten Phase der Adoleszenz sprechen, dann folgen wir damit nicht der Vorstellung, dass die Anfänge und das Ende der Adoleszenz oder zu bearbeitende Themen zeitlich festlegbar sind. Vielmehr gehen wir mit King davon aus, dass ,die immer wieder aufgeworfene Frage, wann die Adoleszenz in modernisierten Gesellschaften endet, prozessual, dynamisch und auf die Qualität der Prozesse der Individuation und der Aneignung von Generativität bezogen beantwortet werden“ muss (2013, S. 244, Herv. i.O.). Die Auseinandersetzung mit dem familialen Erbe, dem eigenen Geworden-Sein und Werden und somit auch den Selbst- und Weltverhältnissen ist jedoch von bestimmten Voraussetzungen und sozialen Bedingungen abhängig und dabei ist, wie Helsper et al. (2018, S. 27) erläutern, eine Prozessdynamik zu beachten: „Während die Frühadoleszenz eher durch erste Formen spontanen, verändernden Handelns und unspezifische Reflexionen gekennzeichnet ist, sind die Phasen systematischeren Erkundens des Neuen, ersten Bewährungen des Neuen und weiterer Handlungserprobungen eher für den weiteren Adoleszenzverlauf anzunehmen. Die Bewährung des Neuen in generalisierten sozialen Zusammenhängen und dessen biographische Reflexion ist eher der Spätadoleszenz zuzuweisen.“.
} 
innen im Alter von dreizehn bis achtzehn Jahren und Familiengespräche werden hier ebenfalls mit der Objektiven Hermeneutik ausgewertet.

Die zwei zuletzt genannten Untersuchungen fokussieren mit der Objektiven Hermeneutik im Vergleich zur ersten Studie von Anfang an auf eine Analyse der Positionierung des Subjekts zu seinem Bildungsverlauf. In der Studie „Die Mühen des Aufstiegs" haben wir mit der Kategorie des Bildungsselbst die im sozialen, familialen und adoleszenten Formierungsprozess entstandene Haltung zu bildungsrelevanten Fragen in der Aneignung des Bildungsaufstiegs beschrieben. Im Projekt „Trübas“ wird die institutionelle Triade aus Adoleszenten, Familie und Klinikschule/ Jugendpsychiatrie hinsichtlich der Modi der Bearbeitung und der damit verbundenen strukturellen Krisenkonstellationen beleuchtet, die mit der räumlichen Trennung von der Familie und dem Umstieg in die Klinikschule verbunden sind.

Mit den gewählten und nach wie vor selten durchgeführten qualitativen Längsschnitten können gegenüber der Messung von merkmalbezogenen Differenzen und Übereinstimmungen zwischen den Erhebungszeitpunkten in quantitativen Längsschnitten (z. B. eines Absinkens oder eine Zunahme der Lernmotivation) die subjektiven Bedeutungszuschreibungen und Verarbeitungsformen schulischer Erfahrungen in der Familie und die adoleszenten Entwicklungs- und Bildungsprozesse in ihren - auch gebrochenen - Sinnzusammenhängen hypothesenbildend in den Blick genommen werden (Nittel 1992, S. 16-18). Gegenüber qualitativ-retrospektiven Einfach- bzw. Querschnitterhebungen ermöglichen die qualitativ-rekonstruktiven Längsschnitte eine kontrastierende Rekonstruktion der Handlungsorientierungen und situativen, wie auch biographischen Deutungen von Ereignissen und Erfahrungen in der Schulkarriere durch den Einbezug weiterer subjektiver Zeitperspektiven mit früheren Narrationen und Entwürfen. Eröffnet wird dadurch ein Zugang, (Um-)Deutungen, neue Entwürfe und Handlungsmuster der Akteur/innen und somit ihr biographisches Werden im Verhältnis von Transformation und Reproduktion zu verstehen, womit das, was Kontinuität auch angesichts von Diskontinuität in Bildungsverläufen und -prozessen zu stiften vermag, im zeitlichen Verlauf herausgearbeitet und somit in besonderer Weise mitvollzogen werden kann (Thiersch 2020b).

\section{Subjekt, Bewährung und Sozialisation: Theoretische Anschlüsse}

In der vergleichenden Interpretation der Ergebnisse der drei Untersuchungen hat sich der Bewährungsbegriff als fruchtbar erwiesen, um die subjektiven Integrationsund Bearbeitungsleistungen in Bildungsverläufen im Rahmen der (milieuspezifischen) Orientierungen und Anforderungen in den jeweiligen Sozialisationsräumen und bezogen auf adoleszente Krisendynamiken theoriesprachlich genauer klären zu können. Wir schließen hier an subjekttheoretische Überlegungen von Oevermann (2004, 2009) und Zizek $(2015,2020)$ an, wenngleich die Strukturlogik der Bewährung des Neuen in und zwischen Familie und Schule in der Adoleszenz bislang nicht in diesen Arbeiten untersucht wurde und Eingang in Bewährungskonzepte gefunden hat.

Bewährung wird aus einer strukturtheoretischen Sozialisationsperspektive in säkularisierten und rationalisierten modernen Bildungs- und Berufswelten zur Sinn- 
quelle für Selbstverwirklichung. Danach muss sich jedes Subjekt einem universalen Bewährungsproblem in Form von lebenspraktischen, diesseitigen Sinn- bzw. Identitätsfragen stellen (Oevermann 1995). Die Adoleszenz ist als der zentrale Ort der Bewährung des Subjekts zu bestimmen (Oevermann 2009, S. 41). Bewährungsanforderungen sind aus psychoanalytischer, struktur- und entwicklungstheoretischer Sicht so in einem grundlegenden Sinne mit der potenziellen zweiten Individuierungschance der Entstehung des „Neuen in der Adoleszenz“ (Oevermann 2004; King 2013) verbunden und auf die Transformationsdynamik in der Auseinandersetzung mit dem familialen Erbe $\mathrm{zu}$ beziehen. In dieser Phase sind Positionen zum beruflichen und sozialen Status zu erarbeiten und diesbezügliche Entscheidungen in Schule, Beruf und Studium zu treffen (Oevermann 2004). Jugendliche setzen sich mit den Haltungen und Praktiken der Eltern und weiterer Bezugspersonen auseinander und verorten sich zu diesen angeeigneten Haltungen zwischen den Polen einer Anknüpfung und Abgrenzung nun selbst (Helsper 2014, S. 141). In dieser Selbstpositionierung können sie sich nicht auf Routinen und bewährte (Krisen-)Lösungen stützen. In dieser „Logik der Bewältigung von Krisen“ (Oevermann 2009, S. 36) eröffnen und vollziehen sich aus dieser Perspektive Bildungsprozesse. Bewährung ist somit konstitutiv als eine relationale Kategorie aufzugreifen. Bewährungsprozesse und konkretes Bewährungshandeln sind in soziale Anerkennungspraxen, pädagogische Generationsbeziehungen und gesellschaftliche Ordnungen und Entwicklungen (z.B. der Bildungsexpansion) sowie damit verbundene Normierungen (dazu auch Wiezorek 2017, S. 28 f.), d.h. auch angelegte „Bewährungsmaßstäbe“ (Behrend und Zizek 2019, S. 13), eingebettet. Entscheidend ist aber, dass die Strukturlogik der Bewährung auf einen dynamischen und offenen Prozess verweist, in dem die soziale Anerkennung eigener Handlungsentwürfe in Familie und Schule eine zentrale Stellung für identitätsstiftende Bewährungsprozesse der Jugendlichen einnimmt. Diese sind damit konfrontiert, Erwartungen von Familie und Schule in Hinblick auf die eigenen Bildungs- und Berufsentwürfe zu bewerten und unabhängig davon, ob sie diese übernehmen oder ablehnen, für sich die (neuen) Handlungsorientierungen zu begründen und zu erproben.

\section{Bewährungsanforderungen in der Adoleszenz zwischen Familie und Schule: Übergreifende Ergebnisse}

Grundsätzlich bestätigen die hier betrachteten qualitativen Längsschnittstudien Fends Analysen (1997, S. 2f.), dass der Umgang mit Schule in der Adoleszenz um zwei Themenbereiche zentriert ist: die Ausbildung eines „Habitus der Aufgabenbewältigung“ (Lern- und Leistungsmotivation bzw. -bereitschaft) und die „Selbstfindung bzw. Identitätsfindung des Menschen in der Moderne“. Während Fend proaktive und interpretative Bewältigungs- und Bearbeitungsprozesse des Subjekts beschreibt, verweisen unsere Ergebnisse darauf, wie die soziale Konstitution des Subjekts in der Dynamik zwischen Familie und Schule spezifische Bewährungsanforderungen impliziert. Im Kern wird mit dem Bewährungsbegriff herausgearbeitet, wie sich junge Menschen in der Adoleszenz gegenüber den schulischen Anforderungsstrukturen vor dem Hintergrund fallspezifischer biographischer Dynamiken, 
familialer Adressierungen und adoleszenter Transformationsprozesse positionieren und positioniert werden. So muss ein ,innerer Zusammenhang zwischen familialer Sozialisation, Selbstgenese und biographisch selektierten Ausformungen individueller Habitusformationen und Bearbeitungsstrategien der Selbstkrisen als Voraussetzungen für die Ausformungen eines schulbiographischen Passungsverhältnisses“ in der Adoleszenz gesehen werden (Kramer 2002, S. 227).

\subsection{Bewährungsdynamiken zwischen Familie und Schule in unterschiedlichen Adoleszenzphasen}

In der vergleichenden Analyse der drei Längsschnittstudien können wir Bewährungsdynamiken in adoleszenten Bildungsprozessen ausdifferenzieren: In der Frühadoleszenz verweisen Ergebnisse - im Vergleich zur 4. und 5. Klasse - auf eine Spannung zwischen den relativ stabilen schul- und bildungsbezogenen Orientierungen der Familie und, infolge der erhöhten schulischen Anforderungen und Erwartungen in Klasse 7, auf distanziertere Schulhaltungen bei Jugendlichen im Verlauf der Sekundarstufe I. Angesichts dieser gesteigerten Anforderungen leistungs- und aufgabenbezogener Bewährung reiben sich erhöhte Erwartungen an die Schüler/ innen mit neuen peerkulturellen Orientierungen am Beginn frühadoleszenter Perspektivenwechsel und Neukonstruktionen in der Distanzierung von erwachsenen Bezugspersonen (Kramer et al. 2013). Insofern bestätigen die Ergebnisse ein Absinken des positiven Schulbezugs in der Leistungs- und Anstrengungsbereitschaft und im Wohlbefinden sowie den Anstieg von Leistungsangst der Schüler/innen zwischen dem 6. und 10. Schuljahr und insbesondere die Relevanz der 7. Jahrgangsstufe ,als kritisches Jahr“" (Fend 1997, S. 182 und S. 247). Dies führt in den Familien zu erhöhtem Konfliktpotential, da auf das eigene Kind projizierte familiale Bildungsprojekte und -orientierungen in Frage gestellt werden; gerade auch wenn die Leistungen der Kinder die familialen Erwartungen übertreffen (z. B. im Schulaufstieg). Jugendliche stehen hier sowohl zwischen einer für sie neuartigen Leistungsbewährung in der Schule als auch einer fallspezifisch konstellierten Bewährungssituation im Kontext familialer Bildungsentwürfe.

In der mittleren Adoleszenz haben Jugendliche im schulischen Raum tendenziell sich bewährende Handlungsroutinen entwickelt und die schulischen Selbstentwürfe wieder stabilisiert. Vor dem Hintergrund des zunehmenden Drucks von anschlieBenden Bildungs- und Berufsentscheidungen nehmen aber die Aushandlungen und Legitimierungen biographischer Bildungs- und Berufsentwürfe in der familialen Interaktion und einer damit verbundenen Anforderung einer jugendlichen Selbstpositionierung zu. Bildungsbiographische Entwürfe werden hier im Kontext familialer Beziehungsstrukturen ausgehandelt (Silkenbeumer et al. 2017). Probleme im Verlauf der Sekundarstufe II stehen daher auch im Zusammenhang mit ungelösten Beziehungs- und Individuationsfragen (Labede et al. 2020). Die Schule stellt sich für die Jugendlichen in dieser Phase dagegen als ein verlässlicher - wenn freilich auch nicht konfliktfreier - Handlungsort dar.

Im Übergang zur Spätadoleszenz artikulieren Jugendliche massiv erfahrene Veränderungen und Anforderungen im Einmünden in die Ausbildung bzw. das Studium. Sie müssen sich im Übergang in die berufliche Ausbildung unmittelbar handlungs- 
praktisch in einem neuen institutionellen Feld bewähren. Damit sind aber ausbildungsbezogene und berufliche Entscheidungen und - wie die Studie „Die Mühen des Aufstiegs“ zeigt - neue Probleme in der Einmündung aber auch in der Familie zu legitimieren. Andererseits konnten wir in allen Untersuchungen auch Einzelfälle jugendlicher Bildungsverläufe nachzeichnen, in denen die Schüler/innen dauerhaft verunsichert sind und keine schulische Identifikation aufbauen. Hier erweist sich vielmehr der Übergang in die Arbeitswelt und die Bewährung in einer Ausbildung als anschlussfähig für das Subjekt (Labede et al. 2020).

\subsection{Bewährungsprozesse Heranwachsender zwischen Individuation und Generativität}

Übergreifend bringen die Ergebnisse Bewährungsprozesse in der Selbstpositionierung zum eigenen Bildungsverlauf der Jugendlichen zum Ausdruck. Idealtypisch stellt sich die Frage, ob und wie der Bildungsverlauf im bisher ausgebildeten, gleichwohl unabschließbaren bildungsbiographischen Selbstentwurf (neu) integriert bzw. desintegriert ist. „Bewährungsfiguren“ der Schüler/innen sind in ihrer biographischen Genese zu verstehen und werden ,nicht über eine explizite Reflexion gebildet“ (Behrend und Zizek 2019, S. 14). Die Eigendynamiken adoleszenter Selbstpositionierungen, familialer und schulischer Interaktion in der Dialektik von Individuation und Generativität (King 2013) müssen so zur Erklärung von Bildungsverläufen und ihrer Deutung jenseits der Gesetzmäßigkeiten im sozialen Raum herangezogen werden, wie vor allem in den Verlaufsformen des Auf-, Ab- und Umstiegs deutlich wird. Die „Mühen des Aufstiegs“ sind so nicht als Mühen zu verstehen, die sich durch den Aufstieg im sozialen Raum und damit verbundene Entfremdungsprozesse ergeben. Vielmehr sind mit dem Aufstieg Bewährungsprozesse der Formierung und Konturierung eines Bildungsselbst verbunden, deren spezifische Ausformung wesentlich auf familiale Binnenverhältnisse (Beziehungsdynamiken, Familienmilieu) in der Auseinandersetzung mit Schule und Bildung zurückzuführen sind (Labede et al. 2020).

Bewährung in Bildungsverläufen impliziert unseren Ergebnissen nach eine Auseinandersetzung mit dem familialen Erbe und damit eine Positionierung in familialen Dynamiken der Konkurrenz, des Übertreffens, des Mithaltens oder des Nachziehens in inter- und intragenerationalen Beziehungen (Eltern-Kind-Beziehungen, Geschwister), die gerade auf schulische Entwicklungen (Bildungserfolg bzw. -misserfolg) rekurrieren. Wie in den Interviews deutlich wird, gestalten sich adoleszente Bildungsverläufe dabei als zu bewältigende Aufgabe für die gesamte Familie, da auf struktureller Ebene Um- und Neugestaltungen von Eltern-Kind-Beziehungen erforderlich werden. Eltern stehen dabei ebenso vor einem Trennungs- und Ablösungsproblem von der eigenen generationellen Position, da auch sie sich mit eigenen (nicht) realisierten Lebensentwürfen in der Konfrontation mit den berufsbiographischen Entscheidungen ihrer Kinder auseinandersetzen müssen (King 2013).

Selbst bei objektiv erfolgreichen Schülern/innen und bei stabilen und sich reproduzierenden habituellen Haltungen in der Familie stellen sich diese Fragen der Positionierung zu zukünftigen Bildungsentwürfen, wie wir sie beim Aufstiegstypus des „Durchhaltens“ bzw. „Durchkommens“ rekonstruieren konnten (Labede et al. 2020). 
Entlang der Befunde der drei Studien zeigt sich somit, dass auch im Bildungserfolg und in der Statussicherung von einem bisher kaum erforschten Bewährungsproblem des Subjekts auszugehen ist. Jenseits des objektiven Bildungserfolgs müssen schul- und bildungsbezogene Ablösungs- und Individuationsprozesse in Beziehungsdynamiken und damit verbundene subjektive Anstrengungen im Positionsgeschehen bearbeitet werden. Bspw. befinden sich Jugendliche aus akademischen Milieus in einer Bewährungskonstellation, in der nur die besten Leistungen (z. B. die perfekte Eins ohne Punktabzug) in den Blick kommen und ein Verfehlen ein „Leiden“ auf hohem Niveau bedeutet (Kramer et al. 2013). Besonders aber der ausbleibende Bildungserfolg und die Nicht-Umsetzung aufstiegsorientierter und strebsamer Bildungsorientierungen der Eltern können zu psychosozialen Belastungen der Schüler/ innen führen. Diese Fallkonstellationen können sich in der frühen Adoleszenz zuspitzen und sowohl in familiale Konflikte als auch schuldistanziertere Orientierungen münden (Kramer et al. 2009, 2013).

Umgekehrt stoßen wir empirisch auf Konstellationen, in denen durch familiale Problemkonstellationen und Krisen belastete Jugendliche sich den schulischen Raum in einer Weise aneignen, die Individuierungsprozesse freisetzt und einer Umgestaltung und Umdeutung des familialen Erbes zuarbeiten kann. Dies ist mit der Bindung an schulische Inhalte und einer Identifikation mit dem schulischen Ordnungsprinzip Leistung verbunden, was jedoch gerade aufgrund von Loyalitätskonflikten auch zu gesteigerten Anstrengungsfiguren beiträgt. Diese Integration des Schulischen in den eigenen Selbstentwurf wird durch die stabilisierende Anerkennung und Adressierung des Jugendlichen in Familie und Peer-Group als besonders leistungsfähig gestützt (Labede et al. 2020, S. 197 f.). Schließlich wird Schule aber auch kompensatorisch als „Zufluchtsort“ und Entlastung gegenüber den familialen Belastungen in der Adoleszenz wahrgenommen. Diese Schüler/innen deuten die Schule und die schulische Leistungsbewährung eher als eine Chance denn als Druck, was kehrseitig eine Daueranstrengung produziert. Diese aus schulfernen Milieus stammenden Schüler/innen müssen sich in einer für sie „fremden“ kulturellen Welt mit guten Leistungen und angepasstem Verhalten beweisen. Bei diesen beiden Typen ermöglicht die Schule den Jugendlichen eine Individuation und Abgrenzung, sodass die Mühen und Kosten einer Habitustransformation in Kauf genommen werden. Empirisch wird übergreifend deutlich, dass die Adoleszenz die Bewährungsphase für die Habitusbildung darstellt, weil Jugendliche sich sowohl in der identifikatorischen Bezugnahme auf Schule als auch in der schulischen Abgrenzung mit dem Habitus der Eltern auseinandersetzen müssen (Helsper 2014; Thiersch 2020a).

\subsection{Bewährung und Übergänge im Schulsystem}

Abschließend möchten wir die in allen Untersuchungen im Zentrum stehenden Übergänge und ihre Bewältigung im Kontext subjektiver Bewährungsanforderungen deuten. Im Vergleich der Ergebnisse zeigt sich eine Asynchronität von subjektiver Bewährung und institutionellem Vollzug von Übergängen. Für das Subjekt relevante und artikulierte Übergänge korrespondieren nicht per se mit den institutionellen Übergangspassagen, wie etwa die Schulwahl oder das Ankommen an einer neuen Schule. Aus subjekttheoretischer Perspektive stellen sich in der Aus- 
einandersetzung mit der Institution Schule und in der Konstruktion von Übergängen Fragen der Selbstpositionierung nicht zwingend - wie lange in der Schulforschung bzw. -pädagogik angenommen - an den institutionellen Passagen als „Übergangsschock" dar (kritisch dazu auch Tillmann 2013). Erst mit der Notwendigkeit, sich in neuen Feldern und angesichts gesteigerter Ablösungsfragen bspw. nach einem Schulformübergang an ein Gymnasium zu bewähren, werden Irritationen, Anpassungsprobleme und Diskontinuitäten im Bildungsverlauf sichtbar (Labede 2019). In den Längsschnitten kann so beobachtet werden, wie positiv gedeutete Erfahrungen zu Beginn an einer neuen Schule in der retrospektiven Betrachtung mitunter negativer bewertet werden. Umgekehrt relativieren sich zu Beginn erfahrene Probleme zu späteren Zeitpunkten oder kommen gar nicht mehr zum Ausdruck. Aus der subjektiven Perspektive äußern sich somit sowohl positiv wahrgenommene Wendepunkte im Bildungslauf aber auch Passungs- und Anerkennungsprobleme unabhängig der institutionellen Übergangsorte (z. B. nach der Grundschule, Sek I und II) mitunter verzögert (Kramer et al. 2013).

Darüber hinaus machen unsere Befunde deutlich, dass - neben Familie und Schule - auch die Peers, Professionelle und weitere Institutionen für Bewährungsfragen in „Übergangsräumen“ und Bildungsverläufen relevant werden können. Dies ist vor allem dann der Fall, wenn institutionelle Akteur/innen über schulbiographische Verläufe als Paten bzw. Gatekeeper für Bildungsaufstiege (Kramer et al. 2013; Silkenbeumer et al. 2017) oder als „Administratoren bzw. Sachverwalter“ im Schulwechsel nach einem Klinikaufenthalt mitentscheiden (Hornung und Becher 2019). In der Institution Klinikschule müssen sich Jugendliche im spannungsvollen Verhältnis von Patienten- und Schülerrolle positionieren. Die in diesem Kontext aufgeworfenen Transformationsanforderungen bzw. -imperative und ein damit verbundener Bewährungsdruck zielen auf die gesamte Konstitution des Subjekts, das sich zwischen Familie, Psychiatrie und Klinikschule (Silkenbeumer et al. 2020) beweisen muss.

\section{Schulische Bildungsverläufe, adoleszente Individuierung und Bewährungsprozesse: Theoretisierung der Ergebnisse}

Schule als bedeutsamer sozialisatorischer Erfahrungsraum lässt sich als das zentrale Bewährungsfeld für Jugendliche, gerade bezogen auf die sozialen Dimensionen Leistung und Status, in modernisierten Gesellschaften betrachten (Flitner 2001). Die präsentierten Ergebnisse zeigen, in welcher Weise und unter welchen Bedingungen Schüler/innen den mit ihren Bildungsverläufen verbundenen Erfahrungen und Anforderungen in Schule und Familie begegnen. Damit werden Fragen nach ihrem kontinuierlichen bildungsbiographischen Werden, den Entwicklungsprozessen und ihren Selbstverortungen sowie nach der Dynamik, die sich im Rahmen transformatorischer Prozesse mit der Integration und Bewährung des Neuen verbindet, umschlossen. Im Folgenden werden die empirischen Befunde zur Bewährung in der Adoleszenz zwischen Familie und Schule dahingehend diskutiert, welche theoretischen Implikationen sie für die Beschreibung von Bildungsprozessen und -karrieren aufweisen. 
Mit der in heuristischer Absicht hier in Umrissen vorgeschlagenen bewährungstheoretischen Perspektive erweist sich die zu beantwortende Frage nach den Formen der Aneignung und Integration des Neuen in der Adoleszenz als bedeutsam. Damit angesprochen ist der Prozess der Formierung des Bildungsselbst im Sinne einer kontinuierlichen Entwicklung bildungsbiographischer Identität (Silkenbeumer und Wernet 2010; Wernet 2012, S. 198 ff.). Denn empirisch stoßen wir nicht nur in aufsteigenden Schulkarrieren (Labede 2019) und - anders gelagert - absteigenden Schulkarrieren (Niemann 2015) und Bildungsverläufen (Schmeiser 2003) mindestens auf Irritationen bisher ausgebildeter Modi der Krisenbearbeitung und Welt- und Selbstentwürfe, die Positionierungen zum bisherigen und antizipierten weiteren Bildungsverlauf im Zuge dieser bildungsbiographischen Einschnitte notwendig werden lassen.

Die Ergebnisse machen so deutlich, dass sich die Auseinandersetzung mit dem kulturellen und sozialen Erbe bzw. dem familialen „Habituserbe“ (Helsper 2014, S. 152) - gerade auch in der Schule und bezüglich der beruflichen Zukunft - in der Adoleszenz in besonderer Weise stellt, weil in dieser Phase die zu bearbeitenden intergenerationalen und familialen Umgestaltungen an Relevanz gewinnen. Diese Beziehungsdynamiken vollziehen sich zwischen einer Anknüpfung an die und Abgrenzung zu den familialen Orientierungen und Praktiken. Die Bewährungsdynamik ist vor dem Hintergrund dieser intersubjektiven und auch intrapsychischen Bedingungen konstelliert. Denn wie sich Heranwachsende gegenüber den an sie familial herangetragenen Erwartungen bis hin zu „Aufträgen“ und Delegationen etwa hinsichtlich der Fortsetzung nicht realisierter „Bildungsprojekte“ ihrer Eltern oder eines Elternteils positionieren können, hängt auch davon ab, in welcher Weise die Umgestaltung intergenerationaler Beziehungen ermöglicht und somit adoleszente Transformation familial bearbeitet wird (King 2013, 2015). In diesem Zusammenhang tritt die Frage nach der Herausbildung von Bewährungsfiguren, der Suche nach und dem Druck der Bewährung im Verhältnis von Schule und Familie in mehrfacher Hinsicht hervor. Wenn es um die Analyse der Konstitution von Bildungsprozessen und mithin von Subjektivität und bildungsbiographischer Identität geht, muss die Frage beantwortet werden, was dem Habitus in der Alltagspraxis seine subjektive Gestalt verleiht. Damit ist auf Prozesse sozialer Binnendifferenzierung und eigenlogischer Interaktionsdynamiken im Verhältnis von Familie und sozialem Milieu zu verweisen (Rademacher und Wernet 2014).

Abschließend lässt sich an die Theorie transformatorischer Bildungsprozesse (Koller 2012) anknüpfend die Relevanz von Bewährungsprozessen weiter pointieren: Denn die in transformatorischen Prozessen im Zuge der Bearbeitung von unerwarteten Problemstellungen in der Auseinandersetzung mit Welt- und Selbstverhältnissen herausgebildeten neuen Entwürfe und Praktiken müssen sich in biographischer Hinsicht - und damit immer in der Beziehung zu anderen - bewähren. In der Bearbeitung der Krise entwickeln sich neue Welt- und Selbstbilder. Nur wenn sich diese auch bewähren können, was nicht zuletzt auf die Bedeutung von Anerkennungsbeziehungen verweist, dann kann u. E. auch von Bildungsprozessen des Subjekts konstitutionstheoretisch ausgegangen werden. So scheint gerade auch für eine an Fragen der jugendlichen Bildungsprozesse ermöglichenden oder verhindernden Konstellationen interessierte sozialisationstheoretisch fundierte biographische und 
intergenerationale Forschungsperspektive die Auseinandersetzung mit bewährungsund bildungstheoretischen Überlegungen fruchtbar. Dabei wären jedoch auch Momente des „Verharren[s]“, „Scheiterns- oder Marginalisierungsprozesse“ (Wiezorek 2017, S. 29) in den Blick zu bringen. Dies bedeutet empirische Antworten auf die Frage zu finden, wie und wodurch Handlungsfähigkeit im Rahmen von Lern- und Bildungsprozessen in Kindheit und Jugend hervorgebracht und aufrechterhalten, aber auch eingeschränkt oder verhindert wird.

\section{Fazit}

Wie eingangs formuliert, sind die sozialisatorischen Dimensionen der Institution Schule und das Dreieck adoleszente Schüler/innen, Schule und Familie ein ,altes" Forschungsfeld sozialwissenschaftlich ausgerichteter Bildungsforschung. Im Zuge gesellschaftlicher und theoretischer Entwicklungen sowie Diskurse des Kompetenzerwerbs, der Leistungsmotivation und Lernoptimierung von Schülern/innen im Aufschwung empirischer Bildungsforschung nahm es in den letzten Jahren jedoch eine marginale Rolle ein. Im vorliegenden Beitrag haben wir Ergebnisse aus Längsschnittstudien der qualitativ-rekonstruktiven Forschung zu Bildungsverläufen zusammenfassend im Blick auf dieses Verhältnis von Familie und Schule sowie adoleszenter Individuierung betrachtet. Im Sinne einer theoretischen Abstraktion konnten wir übergreifend bestimmen, dass sich Jugendliche in adoleszenten Bewährungsprozessen in und zwischen Familie und Schule eigenständig zu ihrem Bildungsverlauf positionieren müssen. Die Befunde verweisen auf für das Subjekt relevante Bewährungsanforderungen, -räume und -phasen. Bewährung kommt damit als ein heuristisches Konzept einer gegenstandsbezogenen Theorieentwicklung in den Blick.

Die sozialisatorische Dimension der Institution Schule und ihre Bedeutung für adoleszente Individuierungsprozesse erweist sich nun gerade darin als relevant, dass sie einen der zentralen Resonanzräume im Sinne eines Orts des In-Sich-BeziehungSetzens zur Welt und zum eigenen Selbst für Jugendliche darstellt (Rosa 2018, S. 402f.). Auch Fend stellt heraus: „Die Schule müsste ein Bildungswesen für Adoleszente sein. Sie müsste Jugendliche auf dem Wege ihrer Identitätsfindung begleiten und könnte durch abgestimmte Bildungsangebote diese persönlichen Entwicklungswege bereichern“ (Fend 1994, S. 8). Ohne auf die damit verbundene implizite Schulkritik einzugehen, lassen sich mit dem hier vorgeschlagenen Konzept der Bewährungsanforderungen des adoleszenten Selbst zwischen Familie und Schule, die empirisch erst in konkreten Handlungsvollzügen und -entwürfen als solche zu entziffern sind (Behrend und Zizek 2019, S. 16), die Konstitutions- und Transformationsprozesse jugendlicher Subjektivität, bildungsbiographischer Positionierungen und Bildungskarrieren subjekttheoretisch ausschärfen. In einem umfassenderen Sinne verweisen diese auf Konstitutionsprozesse des subjektiven Habitus, die im Rahmen von Anerkennungsbeziehungen primär familial hervorgebracht werden (Helsper 2014).

Bewährungsdynamiken und so auch Autonomisierungsanforderungen oder auch -zumutungen in schulischen Bildungsverläufen und adoleszenten Bildungsprozes- 
sen sind also nicht unabhängig von dem zu begreifen, was mit dem Begriff der „Generativität“ näher umschrieben werden kann. Damit wird, wie Vera King (2020, S. 49) erläutert, ,eine vorwiegend produktive Haltung, Wirkmächtigkeit oder Sorge“ analytisch gefasst, wodurch eine dynamische Perspektive auf die „sozialen und generativen Voraussetzungen für jugendliche Entwicklung“ eingenommen werden kann. Angesprochen sind insofern die Chancenstrukturen und die Qualität der Adoleszenz, ,,sodass auch Bedingungen differenziert werden können, unter denen soziale Ungleichheiten, aber auch familiale Konflikte und unbewältigte Themen über Adoleszenzverläufe sich reproduzieren oder eben transformieren“ (King 2020, S. 49). Gerade die Erforschung familialer Interaktionen erweist sich hier als fruchtbare Ergänzung bildungsbiographischer Interviews und schulischer Interaktionssituationen, in denen ferner die Bedeutung von Peerbeziehungen neben Lehrer-Schüler-Beziehungen $\mathrm{zu}$ berücksichtigen wäre. Aus einer jugendtheoretisch akzentuierten bildungswissenschaftlichen Forschungsperspektive wären Sinn(re-)konstruktionen und Selbstverständnisse (dazu Mey 2018) sowie Bewährungsprozesse und -anforderungen deshalb nicht nur im Hinblick auf Schule als ein Raum jugendlicher Lebenswelten zu erforschen und die Schüler/innen als Subjekt in einem umfassenderen Sinne theoriesprachlich und empirisch zu würdigen.

Funding Open Access funding enabled and organized by Projekt DEAL.

Open Access Dieser Artikel wird unter der Creative Commons Namensnennung 4.0 International Lizenz veröffentlicht, welche die Nutzung, Vervielfältigung, Bearbeitung, Verbreitung und Wiedergabe in jeglichem Medium und Format erlaubt, sofern Sie den/die ursprünglichen Autor(en) und die Quelle ordnungsgemäß nennen, einen Link zur Creative Commons Lizenz beifügen und angeben, ob Änderungen vorgenommen wurden.

Die in diesem Artikel enthaltenen Bilder und sonstiges Drittmaterial unterliegen ebenfalls der genannten Creative Commons Lizenz, sofern sich aus der Abbildungslegende nichts anderes ergibt. Sofern das betreffende Material nicht unter der genannten Creative Commons Lizenz steht und die betreffende Handlung nicht nach gesetzlichen Vorschriften erlaubt ist, ist für die oben aufgeführten Weiterverwendungen des Materials die Einwilligung des jeweiligen Rechteinhabers einzuholen.

Weitere Details zur Lizenz entnehmen Sie bitte der Lizenzinformation auf http://creativecommons.org/ licenses/by/4.0/deed.de.

\section{Literatur}

Becher, J., \& Schulmeister, J. (2020). „Ich hab ganz viele Möglichkeiten und die Klinik hat mir wirklich sehr geholfen“ - Die Kinder- und Jugendpsychiatrie als potenzielle Quelle adoleszenter Neuschöpfung. In S. Thiersch (Hrsg.), Qualitative Längsschnittforschung. Bestimmungen, Forschungspraxis und Reflexionen (S. 259-271). Opladen, Berlin, Toronto: Barbara Budrich.

Becher, J., Schulmeister, J., \& Silkenbeumer, M. (2021). Jugendpsychiatrie, Familie und Adoleszente Sozialisationstheoretische Annäherungen an die Figur des institutionellen Dritten als strukturellem Krisenauslöser. Gesellschaft - Individuum - Sozialisation (giso). Zeitschrift Für Sozialisationsforschung, i. E.

Behrend, O., \& Zizek, B. (2019). Einleitung. Zentrale Aspekte von Autonomie und Bewährung für eine rekonstruktive Sozialforschung. In O. Behrend \& B. Zizek (Hrsg.), Autonomie und Bewährung. Grundbegriffe rekonstruktiver Sozialisations- und Bildungsforschung (S. 1-20). Wiesbaden: Springer VS. 
Combe, A., \& Helsper, W. (1994). Was geschieht im Klassenzimmer? Perspektiven einer hermeneutischen Schul- und Unterrichtsforschung. Zur Konzeptualisierung der Pädagogik als Handlungstheorie. Weinheim: Deutscher Studienverlag.

Fend, H. (1994). Die Entdeckung des Selbst und die Verarbeitung der Pubertät. Entwicklungspsychologie der Adoleszenz in der Moderne. Bern: Bernd Huber.

Fend, H. (1997). Der Umgang mit Schule in der Adoleszenz. Aufbau und Verlust von Lernmotivation, Selbstachtung und Empathie. Bern: Bernd Huber.

Flitner, E. (2001). Grundmuster und Varianten von Erziehung in modernen Gesellschaften. Eine erziehungswissenschaftliche Lektüre der herrschafts- und religionssoziologischen Schriften Max Webers. In E. Hanke \& W. J. Mommsen (Hrsg.), Max Webers Herrschaftssoziologie. Studien zur Entstehung und Wirkung (S. 265-284). Tübingen: Mohr Siebeck.

Helsper, W. (2008). Schülerbiografie und Schulkarriere. In W. Helsper \& J. Böhme (Hrsg.), Handbuch der Schulforschung (2. Aufl. S. 903-920). Wiesbaden: Springer VS.

Helsper, W. (2014). Habitusbildung, Krise, Ontogenese und die Bedeutung der Schule - Strukturtheoretische Überlegungen. In W. Helsper, R.-T. Kramer \& S. Thiersch (Hrsg.), Schülerhabitus - Theoretische und empirische Analysen zum Bourdieuschen Theorem der kulturellen Passung (S. 125-158). Wiesbaden: Springer VS.

Helsper, W., Dreier, L., Gibson, A., Kotzyba, K., \& Niemann, M. (2018). Exklusive Gymnasien und ihre Schüler Passungsverhältnisse zwischen institutionellem und individuellem Schülerhabitus. Wiesbaden: Springer VS.

Helsper, W., Kramer, R.-T., Hummrich, M., \& Busse, S. (2009). Jugend zwischen Familie und Schule. Eine Studie zu pädagogischen Generationsbeziehungen. Wiesbaden: Springer VS.

Helsper, W., Müller, H., Nölke, E., \& Combe, A. (1991). Jugendliche Außenseiter. Biographische Rekonstruktionen scheiternder Bildungs- und Ausbildungsverläufe. Opladen: Westdeutscher Verlag.

Hornung, J., \& Becher, J. (2019). Von Förderschule zu Förderschule - Zum Motiv der Entlastung in der Rekonstruktion eines Übergabegesprächs nach dem Aufenthalt in einer Kinder- und Jugendpsychiatrie. In S. Ellinger \& H. Schott-Leser (Hrsg.), Rekonstruktionen sonderpädagogischer Praxis. Eine Fallsammlung für die Lehrerbildung (S. 75-102). Opladen, Berlin, Toronto: Barbara Budrich.

King, V. (2009). Ungleiche Karrieren. Bildungsaufstieg und Adoleszenzverläufe bei jungen Männern und Frauen aus Migrantenfamilien. In V. King \& H.-C. Koller (Hrsg.), Adoleszenz - Migration - Bildung. Bildungsprozesse Jugendlicher und junger Erwachsener mit Migrationshintergrund (2. Aufl. S. 27-46). Wiesbaden: Springer VS.

King, V. (2013). Die Entstehung des Neuen in der Adoleszenz. Individuation, Generativität und Geschlecht in modernisierten Gesellschaften. Wiesbaden: Springer VS.

King, V. (2015). Kindliche Angewiesenheit und elterliche Generativität. Subjekt- und kulturtheoretische Perspektiven. In S. Andresen, C. Koch \& J. König (Hrsg.), Vulnerable Kinder. Interdisziplinäre Annäherungen (S. 23-43). Wiesbaden: Springer VS.

King, V. (2020). Zur Theorie der Jugend. Problemstellungen - Konstitutionslogik - Perspektiven. In A. Heinen, C. Wiezorek \& H. Willems (Hrsg.), Entgrenzung der Jugend und Verjugendlichung der Gesellschaft. Zur Notwendigkeit einer „Neuvermessung“ jugendtheoretischer Positionen (S. 39-53). Weinheim, Basel: Beltz.

King, V., Koller, H.-C., Zölch, J., \& Carnicer, J. (2011). Bildungserfolg und adoleszente Ablösung bei Söhnen aus türkischen Migrantenfamilien. Eine Untersuchung aus intergenerationaler Perspektive. Zeitschrift für Erziehungswissenschaft, 14(4), 581-601.

Koller, H.-C. (2012). Bildung anders denken. Einführung in die Theorie transformatorischer Bildungsprozesse. Stuttgart: Kohlhammer.

Kramer, R.-T. (2002). Schulkultur und Schülerbiographien. Das „schulbiographische Passungsverhältnis “. Opladen: Leske + Budrich.

Kramer, R.-T. (2014). Identität als Passungsverhältnis von Schüler-Selbst und Schulkultur. In J. Hagedorn (Hrsg.), Jugend, Schule und Identität (S. 423-438). Wiesbaden: Springer VS.

Kramer, R.-T., Helsper, W., Thiersch, S., \& Ziems, C. (2009). Selektion und Schulkarriere. Kindliche Orientierungsrahmen beim Übergang in die Sekundarstufe I. Wiesbaden: Springer VS.

Kramer, R.-T., Helsper, W., Thiersch, S., \& Ziems, C. (2013). Das 7. Schuljahr. Wandlungen des Bildungshabitus in der Schulkarriere? Wiesbaden: Springer VS.

Labede, J. (2019). Bildungsbiografische Diskontinuitäten. Zur Krisenförmigkeit von Schulwechseln in der Ado-leszenz. Wiesbaden: Springer VS.

Labede, J., Silkenbeumer, M., Thiersch, S., \& Wernet, A. (2020). Selbstpositionierungen im Bildungsaufstieg. Bildungsselbst, Familiale Dynamiken und adoleszente Transformationsprozesse. In 
S. Thiersch, M. Silkenbeumer \& J. Labede (Hrsg.), Individualisierte Übergänge. Aufstiege, Abstiege und Umstiege im Bildungssystem (S. 185-205). Wiesbaden: Springer VS.

Mey, G. (2018). Jugendforschung: Konjunkturen, Krisen, Konstruktionen. In A. Kleeberg-Niepage \& S. Rademacher (Hrsg.), Kindheits- und Jugendforschung in der Kritik. (Inter-)Disziplinäre Perspektiven auf zentrale Begriffe und Ansätze (S. 273-297). Wiesbaden: Springer VS.

Niemann, M. (2015). Der „Abstieg“ in die Hauptschule. Vom Hauptschülerwerden zum Hauptschülersein - ein qualitativer Längsschnitt. Wiesbaden: Springer VS.

Nittel, D. (1992). Gymnasiale Schullaufbahn und Identitätsentwicklung: eine biographieanalytische Studie. Weinheim: Dt. Studienverlag.

Oevermann, U. (1995). Ein Modell der Struktur von Religiosität. Zugleich ein Strukturmodell von Lebenspraxis und von sozialer Zeit. In M. Wohlrab-Sahr (Hrsg.), Biographie und Religion: Zwischen Ritual und Selbstsuche (S. 27-103). Frankfurt a. M.: Campus.

Oevermann, U. (2004). Sozialisation als Prozess der Krisenbewältigung. In D. Geulen \& H. Veith (Hrsg.), Sozialisationstheorie interdisziplinär (S. 155-181). Stuttgart: Lucius \& Lucius.

Oevermann, U. (2009). Biographie, Krisenbewältigung und Bewährung. In S. Bartmann, A. Fehlhaber, S. Kirsch \& W. Lohfeld (Hrsg.), ,Natürlich stört das Leben ständig“ - Perspektiven auf Entwicklung und Erziehung (S. 35-55). Wiesbaden: Springer VS.

Pongratz, L. A., Reichenbach, R., \& Wimmer, M. (Hrsg.). (2007). Bildung - Wissen - Kompetenz. Bielefeld: Janus.

Rademacher, S., \& Wernet, A. (2014). „One Size fits all“. Eine Kritik des Habitusbegriffs. In W. Helsper, R.-T. Kramer \& S. Thiersch (Hrsg.), Schülerhabitus. Theoretische und empirische Analysen zum Bourdieuschen Theorem der kulturellen Passung (S. 159-182). Wiesbaden: Springer VS.

Rosa, H. (2018). Resonanz. Eine Soziologie der Weltbeziehung. Frankfurt a. M.: Suhrkamp.

Schmeiser, M. (2003). „Missratene“ Söhne und Töchter. Verlaufsformen des sozialen Abstiegs in Akademikerfamilien. Konstanz: UVK.

Silkenbeumer, M., \& Wernet, A. (2010). Biographische Identität und Objektive Hermeneutik: methodologische Überlegungen zum narrativen Interview. In B. Griese (Hrsg.), Subjekt - Identität - Person? (S. 171-196). Wiesbaden: VS.

Silkenbeumer, M., Becher, J., \& Schulmeister, J. (2020). Patient werden und Schüler bleiben. Fallstudie zur bildungsbiografischen Bearbeitung von Übergängen im Schnittfeld von Familie, Jugendpsychiatrie und (Klinik-)Schule. In S. Thiersch, M. Silkenbeumer \& J. Labede (Hrsg.), Individualisierte Übergänge. Aufstiege, Abstiege und Umstiege im Bildungssystem (S. 151-184). Wiesbaden: Springer VS.

Silkenbeumer, M., Thiersch, S., \& Labede, J. (2017). Zur Aneignung des Schulaufstiegs im Kontext adoleszenter Individuation und familialer Interaktion. Diskurs Kindheits- und Jugendforschung, 12(3), 343-360.

Thiersch, S. (2020a). Habitus, Bildung und Bewährung - Anfragen und Differenzierungen zum Konzept der kulturellen Passung von Familie und Schule aus subjekttheoretischer Perspektive. In M. Hermes \& M. Lotze (Hrsg.), Bildungsorientierungen. Theoretische Reflexionen und empirische Erkundungen (S. 25-46). Wiesbaden: Springer VS.

Thiersch, S. (2020b). Qualitative Längsschnittforschung - Bestimmungen, Forschungspraxis und Reflexionen. Farmington Hills: Babara Budrich.

Tillmann, K.-J. (2013). Die Bewältigung von Übergängen im Lebenslauf - eine biographische Perspektive. In G. Bellenberg \& M. Forell (Hrsg.), Bildungsübergänge gestalten. Ein Dialog zwischen Wissenschaft und Praxis (S. 15-31). Münster: Waxmann.

Wellendorf, F. (1969). Zur Situation des höheren Schülers in Familie und Schule. In M. Liebel \& F. Wellendorf (Hrsg.), Schülerselbstbefreiung. Voraussetzungen und Chancen der Schülerrebellion (S. 11-91). Frankfurt a.M.: Suhrkamp.

Wellendorf, F. (1974). Schulische Sozialisation und Identität. Weinheim, Basel: Beltz.

Wernet, A. (2012). Die objektive Hermeneutik als Methode der Erforschung von Bildungsprozessen. In K. Schittenhelm (Hrsg.), Qualitative Bildungs- und Arbeitsmarktforschung. Theoretische Grundlagen und Methoden (S. 183-202). Wiesbaden: Springer VS.

Wiezorek, C. (2017). Biographieforschung und Bildungsforschung: Potenziale erziehungswissenschaftlicher Perspektiven auf die Erforschung von Lern- und Bildungsprozessen. Zeitschrift für Qualitative Forschung, 18(1), 21-40.

Zizek, B. (2015). Der Mensch als Bewährungssucher - Versuch einer systematischen Einführung des Begriffs Bewährung in die Sozialwissenschaft. In D. Garz \& B. Zizek (Hrsg.), Wie wir zu dem werden, 
was wir sind. Sozialisations-, biographie- und bildungstheoretische Aspekte (S. 71-88). Wiesbaden: Springer VS.

Zizek, B. (2020). Adoleszente als Bewährungssucher - Charakteristika, Tendenzen und Probleme im Prozess der Erwachsenwerdens anhand eines internationalen Vergleichs. In A. Heinen, C. Wiezorek \& H. Willems (Hrsg.), Entgrenzung der Jugend und Verjugendlichung der Gesellschaft. Zur Notwendigkeit einer „Neuvermessung“ jugendtheoretischer Positionen (S. 158-175). Weinheim, Basel: Beltz. 